\title{
A Modified General Polarimetric Model-based Decomposition Method with The Simplified Neumann Generalized Volume Scattering Model
}

\author{
Qinghua Xie, Jianjun Zhu, Juan M. Lopez-Sanchez, Senior Member, IEEE, \\ Changcheng Wang, and Haiqiang Fu
}

\begin{abstract}
This letter proposes a modified general polarimetric model-based decomposition method which includes a simplified Neumann generalized volume scattering model (SNVSM). This is useful to avoid a known limitation in one of the state-of-the-art general model-based decomposition methods (i.e. Chen's method), which considers only four possible discrete volume scattering models. Two types of SNVSM, assuming horizontal or vertical dipoles, are derived from Neumann generalized volume scattering model. The resulting volume coherency matrix exhibits a continuous range of volume scattering models. In addition, this volume model covers both random and non-random volume cases, which are distinguished by a randomness parameter. Monte Carlo simulations of polarimetric synthetic aperture radar (PolSAR) data are used to test this approach. The proposed method with SNVSM overall improves the final accuracy of estimated parameters in comparison with the original approach and shows consistency with another existing general volume scattering model (i.e. GVSM). In addition, results from two fully polarimetric C-and L-band AIRSAR images over San Francisco region show the proposed method produces reasonably physical results and outperforms the traditional Y4R method. Finally, the differences obtained between SNVSM and GVSM in two building areas show the potential advantage of SNVSM in identifying more types of volume scenes than GVSM.
\end{abstract}

Index Terms-model-based decomposition, generalized volume scattering model, synthetic aperture radar (SAR), Monte Carlo simulation, radar polarimetry.

\section{INTRODUCTION}

$\mathrm{T}$ rget decomposition of polarimetric synthetic aperture radar (PolSAR) is a useful technique to exploit differences of polarimetric signatures to identify and separate scattering mechanisms in natural media [1]-[3]. In past decades, this technique has been widely used in many different applications, such as land-cover classification, target detection, damage assessment, and soil moisture estimation [4]. Target decomposition mainly includes two categories: coherent decomposition of the measured Sinclair matrix, and incoherent decomposition of the measured coherency/covariance

This work was supported in part by National Natural Science Foundation of China under Grant 41531068, 41371335 and 41274010, Spanish MINECO, AEI and EU ERDF under Projects TIN2014-55413-C2-2-P and TEC2017-85244-C2-1-P, and China Scholarship Council under Grant 201406370079. (Corresponding authors: Jianjun Zhu.)

Q. Xie is with the School of Geosciences and Info-Physics, Central South University, Changsha 410083, China, and also the Institute for Computing Research (IUII), University of Alicante, Alicante 03690, Spain.(e-mail: csuxqh@csu.edu.cn).

J. Zhu, C. Wang and H. Fu are with the School of Geosciences and Info-Physics, Central South University, Changsha 410083, China. (e-mail: zjj@csu.edu.cn; wangchangcheng@csu.edu.cn; haiqiangfu@csu.edu.cn ).

J. M. Lopez-Sanchez is with the Institute for Computing Research (IUII), University of Alicante, Alicante 03690, Spain. (e-mail: juanma.lopez@ua.es). matrix [1]-[4]. Because of the diversity of scattering models and parameter inversion algorithms, model-based decomposition is a very active research line in the incoherent decomposition topic [4]. The general idea of any model-based decomposition is to fit some simple physically-based scattering models to the observations. Freeman-Durden three-component decomposition is the original model-based decomposition method, which considers the presence in the scene of three scattering mechanisms: surface, double-bounce, and volume [5]. Although it has been widely used, some significant limitations have been identified, such as model inversion priority, branch conditions, and negative powers [4]. As a result, many modifications have been proposed in the literature to improve the decomposition performance [6]-[9]. In order to overcome the mentioned limitations, a general model-based decomposition (GMD) approach was proposed recently by Chen et al.[6], which incorporates many advances and key ideas published previously. Starting at Chen's decomposition framework, we proposed in [9] some modifications in the parameter inversion algorithm (hereafter named as "Modified Chen" method) to obtain more physically reliable.

Chen's decomposition framework considers that the volume scattering of the scene should match one of four possible discrete volume scattering models, which are obviously not enough to characterize complex volume scenes. Therefore, this feature affects the decomposition performance. A feasible solution consists in using generalized volume scattering models (i.e. defined in a continuous range of parameters) instead of just four discrete models. All available generalized volume models [10]-[15] are defined by one or more parameters. Consequently, this alternative entails increasing the total number of model parameters (unknowns to be estimated) above the number of observations, hence the inversion becomes an underdetermined problem. To avoid this issue, the unknowns in the generalized volume scattering model should be determined in advance. In the same vein, to ease the inversion, generalized volume scattering models defined with only one parameter are preferred.

Following this argument, two general polarimetric model-based decomposition methods incorporating generalized volume scattering models were proposed recently [16]. The first is called simplified adaptive volume scattering model (SAVSM) [12], which actually is a simplified version of Arii model [10] by considering the distribution function as the n-th sine and cosine functions with zero mean value for horizontal and vertical cases. By doing so, the SAVSM depends only on one unknown parameter rather than two in Arii model. Another one is the generalized volume scattering model (GVSM) [11] proposed by Antropov et al. after considering geophysical media symmetry.

As we will illustrate in this letter, results suggest that the method with GVSM (GMD-GVSM) outperforms both the method with SAVSM (GMD-SAVSM) and the so-called "Modified Chen" method regarding the final accuracy of estimated parameters. However, the coherency matrix employed by GVSM assumes $T 22=T 33$, which is fulfilled only in the case of purely random volume [3], [17]. Consequently, exploitation of generalized volume scattering model without this assumption (i.e. considering also $T 22 \neq T 33$ ) needed 
further investigation. In this letter, we propose a general four-component model-based decomposition method which employs a simplified generalized volume scattering model, the original version of which was proposed by Neumann et al.[14], [15]. The adopted volume scattering model can cover cases with both $T 22=T 33$ and $T 22 \neq T 33$, which are classified by a randomness parameter.

The structure of this paper is organized as follows. In Section II, the Neumann volume scattering model is introduced. Then, a simplified parameter inversion is derived in Section III. Section IV describes the proposed modified general decomposition method by using the simplified generalized volume scattering model. In Section V, Monte Carlo simulation tests and two experiments using real PolSAR images at different frequencies over San Francisco area are presented. Result analysis over different interest areas is also provided. Finally, conclusions are drawn in Section VI.

\section{Neumann Volume Scattering Model (NVSM)}

Assuming particles have an axis of symmetry in the polarization plane, one may give the scattering matrix of the average particle in the eigenpolarizations $\hat{p}_{a}, \hat{p}_{b}$ by [14], [15]

$$
S=\left[\begin{array}{cc}
S_{a a} & 0 \\
0 & S_{b b}
\end{array}\right]=\frac{S_{a a}+S_{b b}}{2}\left[\begin{array}{cc}
1+\delta^{*} & 0 \\
0 & 1-\delta^{*}
\end{array}\right]
$$

where $*$ denotes the complex conjugate operator; $\delta$ is the particle scattering anisotropy, which can be expressed as [14], [15]

$$
\delta=\left(\frac{S_{a a}-S_{b b}}{S_{a a}+S_{b b}}\right)
$$

The particle scattering anisotropy $\delta$ characterizes the effective shape of the average particle. With the variation of $|\delta|$, the particle shape varies from an isotropic sphere $(|\delta|=0)$ to a dipole $(|\delta|=1)$. By normalizing with reference to the first element, the normalized coherency matrix of a single particle can be written as [14], [15]

$$
T_{\delta}=\left[\begin{array}{ccc}
1 & \delta & 0 \\
\delta^{*} & |\delta|^{2} & 0 \\
0 & 0 & 0
\end{array}\right]
$$

Under the central limit theorem condition, Neumann et al. suggest the orientation of volume particles follow a von Mises distribution (also known as the circular normal distribution). Under the linear approximation for the orientation distribution, one obtains two linear models for the coherency matrix form [14]

$$
T_{v}(\delta, \tau)=\left\{\begin{array}{c}
\frac{1}{1+|\delta|^{2}}\left[\begin{array}{ccc}
1 & (1-\tau) \delta & 0 \\
(1-\tau) \delta^{*} & (1-\tau)|\delta|^{2} & 0 \\
0 & 0 & \tau|\delta|^{2}
\end{array}\right] \tau \leq \frac{1}{2} \\
\frac{1}{1+|\delta|^{2}}\left[\begin{array}{ccc}
1 & (1-\tau) \delta & 0 \\
(1-\tau) \delta^{*} & |\delta|^{2} / 2 & 0 \\
0 & 0 & |\delta|^{2} / 2
\end{array}\right] \tau>\frac{1}{2}
\end{array}\right.
$$

where $\tau \in[0,1]$ represents the normalized degree of orientation randomness. The volume can change from the case with a single orientation direction ( $\tau=0)$ to the completely random case $(\tau=1)$.

\section{Simplified Neumann Volume Scattering Model (SNVSM)}

As shown in equation (4), the NVSM depends on two unknown parameters. Therefore, the model should be simplified for enabling parameter inversion. As dipoles are generally valid for forests [13], it is usual to assume this case. Then, we set $\delta= \pm 1$, and consequently, the two coherency matrix forms are derived as

$$
T_{v}^{H-S N V S M}=\left\{\begin{array}{l}
\frac{1}{2}\left[\begin{array}{ccc}
1 & 1-\tau & 0 \\
1-\tau & 1-\tau & 0 \\
0 & 0 & \tau
\end{array}\right] \\
\frac{1}{2}\left[\begin{array}{ccc}
1 & 1-\tau & 0 \\
1-\tau & 1 / 2 & 0 \\
0 & 0 & 1 / 2
\end{array}\right]
\end{array} T_{v}^{V-S N V S M}=\left\{\begin{array}{l}
\frac{1}{2}\left[\begin{array}{ccc}
1 & \tau-1 & 0 \\
\tau-1 & 1-\tau & 0 \\
0 & 0 & \tau
\end{array}\right] \quad \tau \leq \frac{1}{2} \\
\frac{1}{2}\left[\begin{array}{ccc}
1 & \tau-1 & 0 \\
\tau-1 & 1 / 2 & 0 \\
0 & 0 & 1 / 2
\end{array}\right] \tau>\frac{1}{2}
\end{array}\right.\right.
$$

where $T_{v}^{H-S N V S M}$ and $T_{v}^{V-S N V S M}$ are the horizontal and vertical simplified Neumann volume scattering models (i.e., H-SNVSM and V-SNVSM) for dipoles, respectively. $T_{v}^{H-S N V S M}=T_{v}^{V-S N V S M}$ is satisfied when $\tau=1$. It is not difficult to check that this model shows good agreement with the three typical volume scattering models in Yamaguchi-based decompositions. Moreover, according to the different values of $\tau$, it covers cases of both $T 22=T 33$ (for $\tau>1 / 2$ ) and $T 22 \neq T 33$ (for $\tau \leq 1 / 2$ ).

As used in [10], [12], we adopt two scattering randomness measurements (the radar vegetation index RVI, and entropy $H$ ) to perform further analysis of SNVSM. As shown in Fig. 1(a), it is obvious that the values of $R V I$ and $H$ increase monotonically when $\tau$ increases. The values arrive at a peak when $\tau$ is equal to 1 , i.e. the completely random case. As it is known, the co-polarization power ratio $\gamma$ (i.e., $\gamma=\left\langle\left|S_{h h}\right|^{2}\right\rangle /\left\langle\left|S_{v v}\right|^{2}\right\rangle$ ) exhibits various relative magnitudes for PolSAR data and has been used to choose a volume scattering model in Yamaguchi decomposition method. In fact, the three typical cases of volume scattering model in Yamaguchi-based decomposition correspond to three specific co-polarization power ratios (i.e., 8/3, 1, 3/8). Fig. 1(b) presents the dependence of the co-polarization power ratios of H-SNVSM and V-SNVSM on the degree of the orientation randomness $\tau$. It is clear that the SNVSM considers a continuous range of this ratio. Moreover, a null ratio is a suitable threshold to distinguish the H-SNVSM and V-SNVSM and the values of $\gamma$ in both sides vary monotonically as a function of $\tau$.

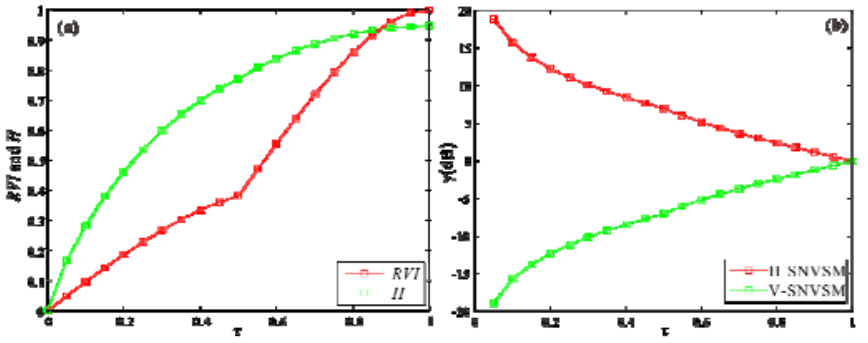

Fig. 1. SNVSM. (a) RVI and Entropy. (b) Co-polarization power ratio

\section{General Model-Based Decomposition}

As it is described in [6], [16], the general four-component model-based decomposition framework can be expressed as

$$
T=f_{v} T_{v}^{G}+f_{s} T_{s}\left(\psi_{S}\right)+f_{d} T_{d}\left(\psi_{D}\right)+f_{c} T_{c}+T_{\text {residual }}
$$

where

$$
\begin{aligned}
T_{\mathrm{s}}= & {\left[\begin{array}{ccc}
1 & \beta^{*} & 0 \\
\beta & |\beta|^{2} & 0 \\
0 & 0 & 0
\end{array}\right] T_{\mathrm{d}}=\left[\begin{array}{ccc}
|\alpha|^{2} & \alpha & 0 \\
\alpha^{*} & 1 & 0 \\
0 & 0 & 0
\end{array}\right] T_{c}=\frac{1}{2} f_{c}\left[\begin{array}{ccc}
0 & 0 & 0 \\
0 & 1 & \pm j \\
0 & \mp j & 1
\end{array}\right] } \\
& T_{s}\left(\psi_{S}\right)=R_{3}\left(\psi_{S}\right) T_{s} R_{3}^{H}\left(\psi_{S}\right), T_{d}\left(\psi_{D}\right)=R_{3}\left(\psi_{\mathrm{D}}\right) T_{\mathrm{d}} R_{3}^{H}\left(\psi_{\mathrm{D}}\right)
\end{aligned}
$$

where $f_{v}, f_{s}, f_{d}$ and $f_{c}$ represent the coefficients of volume, surface, double-bounce and helix scattering, respectively. By introducing two 
independent orientation angles separately, i.e. $\psi_{S}$ and $\psi_{D}$, the generalized surface and double-bounce models proposed by Chen et al. are considered. $T_{v}^{G}$ represents an arbitrary generalized volume scattering model.

In [16], we proposed two general polarimetric model-based decomposition (GMD) methods which incorporate the SAVSM or the GVSM model proposed by Huang et al. and Antropov et al., respectively. The inversion results show GMD-GVSM method is a relatively better general decomposition method. The GMD-GVSM decomposition is expressed as [16]

$$
T=f_{v} T_{v}^{G V S M}(\gamma)+f_{s} T_{s}\left(\psi_{S}\right)+f_{d} T_{d}\left(\psi_{D}\right)+f_{c} T_{c}+T_{\text {residual }}
$$

where $\gamma$ represents the co-polarization power ratio of $\mathrm{HH}$ and $\mathrm{VV}$ components, and $T_{v}^{G V S M}(\gamma)$ represents the coherence matrix of GVSM model, which can be written as [13], [16]

$$
T_{v}^{G V S M}(\gamma)=\frac{1}{\frac{3(1+\gamma)}{2}-\frac{\sqrt{\gamma}}{3}}\left[\begin{array}{ccc}
\frac{1+\gamma}{2}+\frac{\sqrt{\gamma}}{3} & \frac{\gamma-1}{2} & 0 \\
\frac{\gamma-1}{2} & \frac{1+\gamma}{2}-\frac{\sqrt{\gamma}}{3} & 0 \\
0 & 0 & \frac{1+\gamma}{2}-\frac{\sqrt{\gamma}}{3}
\end{array}\right]
$$

where $T 22=T 33$ is inherently assumed in the GVSM model.

Similarly, after substituting the proposed SNVSM (5) into (6), the modified general model-based decomposition method, i.e. GMD-SNVSM, is expressed as

$$
T=f_{v} T_{v}^{S N V S M}(\tau)+f_{s} T_{s}\left(\psi_{S}\right)+f_{d} T_{d}\left(\psi_{D}\right)+f_{c} T_{c}+T_{\text {residual }}
$$

It is not difficult to check that the number of unknowns is ten, whereas the whole coherency matrix can provide only nine real observations. Therefore, similarly to [16], the parameter inside the volume scattering model (i.e. $\tau$ ) should be determined in advance. According to the analysis of SNVSM provided in Section 2, the co-polarization power ratio $\gamma$ has the ability to distinguish the two types of SNVSM and shows high correlation with the degree of orientation randomness $\tau$. Based on it, we propose a criterion to determine $\tau$ by comparing the difference between the ratios $\gamma$ derived by the coherency matrix and by the SNVSM. The optimum $\tau$ is selected when the difference is minimum, i.e.

$$
\min \left|\gamma(T)-\gamma\left(T_{v}^{S N V S M}(\tau)\right)\right|
$$

In addition, in order to reduce the coupling with the volume component of other scattering sources not originated within the canopy, we first apply Lee's polarimetric orientation compensation method [2] and then estimate the corresponding ratio $\gamma$. For every pixel, once $\tau$ is computed, the corresponding volume model is determined according to (7). Afterwards, the remaining nine-unknown equation system is solved by using a nonlinear least-square optimization based on the modified inversion algorithm proposed in [9]. The flowchart of this method is shown in Fig. 2. The steps for determining $\tau$ in GMD-SNVSM are included in the red rectangle.

\section{RESULTS AND DISCUSSION}

\section{A. Monte Carlo Simulations}

We employ the same Monte Carlo simulations of [16] to evaluate the decomposition performance. By selecting sets of values of volume, surface, and dihedral scattering coefficients (i.e. $f_{v}, f_{s}, f_{d}$ ), a total of 216 different scattering scenarios were simulated. Note that the uniformly random dipoles volume model $(T 22=T 33)$ was adopted in simulations. The specific values of parameters are shown in Table I. For comparison, the "Modified Chen" method [9] and the GMD-GVSM method [16] are selected as the reference methods. Fig. 3 shows the cumulative probability distribution curves of root mean square error (RMSE) of all parameters for all cases. Note that higher accuracy is present when the curve arrives at saturation earlier.

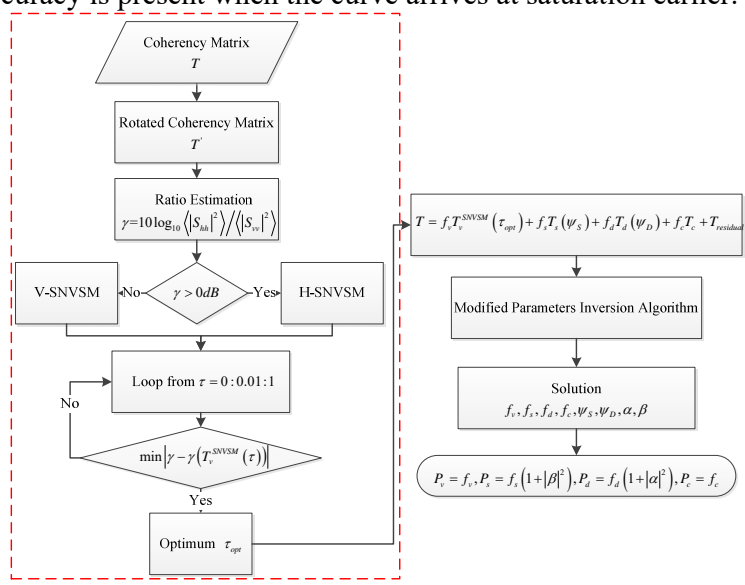

Fig. 2. Flowchart of proposed GMD-SNVSM method.

From Fig. 3, it can be observed that the methods with continuous volume scattering model, i.e. GMD-GVSM and GMD-SNVSM show overall improvements compared with Modified Chen method. Despite they show a little worse performance in retrieving beta (see Fig. 3(i)), they also provide a reasonable estimate since the probability of success in the retrieval is $80 \%$ allowing a 0.08 RMSE value. GMD-SNVSM method presents as good performance as GMD-GVSM method, but with a slight difference in retrieving $f_{v}$ and $f_{s}$.

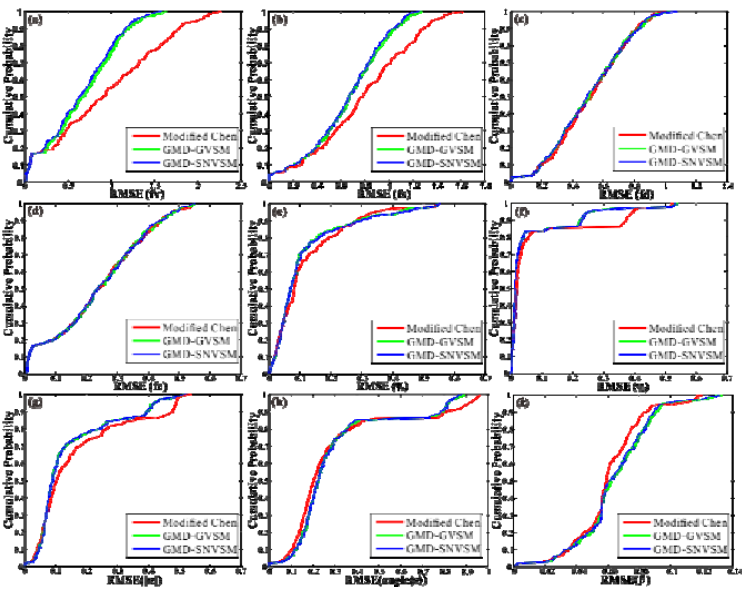

Fig. 3. Cumulative probability distribution curves of root mean square error (RMSE) of all the model parameters from "Modified Chen", GMD-GVSM and proposed GMD-SNVSM methods.

TABLE I

VALUES FOR INPUT PARAMETERS

\begin{tabular}{clc}
\hline \hline \multirow{2}{*}{ Parameter } & \multicolumn{1}{c}{ Quantity } & Value \\
\hline$f_{v}$ & volume scattering coefficient & $0: 2: 10$ \\
$f_{s}$ & surface scattering coefficient & $0: 2: 10$ \\
$f_{d}$ & dihedral scattering coefficient & $0: 2: 10$ \\
$f_{c}$ & helix scattering coefficient & 0.01 \\
$\psi_{s}$ & orientation angle in surface scattering & $-10^{\circ}$ \\
$\psi_{d}$ & orientation angle in dihedral scattering & $-15^{\circ}$ \\
$\alpha$ & ratio parameter in dihedral scattering & $0.3515-0.0768 \mathrm{i}$ \\
$\beta$ & ratio parameter in surface scattering & -0.3377
\end{tabular}




\begin{tabular}{llc}
$\theta$ & incidence angle & $45^{\circ}$ \\
$\phi$ & differential propagation phase & $10^{\circ}$ \\
$\varepsilon_{s}$ & soil dielectric constant & 10 \\
$\varepsilon_{t}$ & trunk dielectric constant & 30 \\
\hline
\end{tabular}

\section{B. Real data test}

Two AIRSAR fully polarimetric images acquired on May 11, 1999 at C- and L-band, respectively, over San Francisco, CA, USA (Lat/Lon, $\mathrm{N} 37.78^{\circ} / \mathrm{W} 122.18^{\circ}$ ) were employed for validation. The original range and azimuth pixel spacing are $3.3 \mathrm{~m}$ and $9.3 \mathrm{~m}$. A multi-look processing with 3 azimuth looks was applied for obtaining near-square pixels and further speckle reduction. Finally, the size of whole image is $1168 \times 843$ pixels and the corresponding incidence angle range is wide, varying from $28.40^{\circ}$ to $62.68^{\circ}$.

In addition to the three general decomposition methods (i.e. Modified Chen [9], GMD-GVSM [16] and GMD-SNVSM), Yamaguchi decomposition with orientation compensation (Y4R) was also adopted as a comparative approach. For our case, it is difficult to identify differences among them with a visual inspection, and all results present a similar trend. Therefore, only the color-coded scattering power decomposition images from GMD-SNVSM method at two bands are used to describe their performances in different land cover types, as shown in Fig. 4. We can see that the volume scattering is dominant in forest and park areas, hence the color is green. Surface scattering is dominant in ocean areas; hence the color is blue. The built-up area shows overall red because of a dominant double-bounce scattering, except for the triangle area located in the right-middle of the images. It means the polarimetric orientation compensation (also named deorientation processing) in all methods is not enough to compensate the overestimation of volume scattering in this area.

For further comparison, the average scattering power proportion of five patches from Fig. 4(a) and Fig. 4(b) including forest area, park area, largely oriented build-up area (Building A), small oriented build-up area (Building B) and ocean area are shown in Table II (C-band) and Table III (L-band). Note that we just make a comparison of different methods and provide some physical explanations, because it is still a difficult issue to quantitatively validate which method shows more consistence with ground truth. In forest areas, a dominating volume scattering is observed at C-band for all methods and all three general methods somehow show higher double-bounce scattering than Y4R method. One possible explanation is that the tested forest area is a part of Mount Sutro forest in which about $80 \%$ is made up of eucalyptus trees exhibiting a large trunk but a low-density crown. For this kind of structure, double-bounce scattering from ground-trunk structure is expected to appear. Moreover, this phenomenon will be more obvious for long wavelength radar. From Table III, we can see that the double-bounce scattering in this forest area is stronger at L-band as expected, but the volume scattering is still dominant. For the park area at C-band, the volume scattering dominates in Y4R, GMD-GVSM and GMD-SNVSM methods. However, the double-bounce scattering dominates in Modified Chen method, but there is still existing strong volume scattering. A possible explanation is that only Modified Chen method incorporates an entropy model, which entails a lower volume component since $\mathrm{Pv}$ reaches a value of $6\left\langle\left|S_{h v}\right|^{2}\right\rangle$ at most. Another phenomenon that can be observed is that the Modified Chen method always provides lowest volume scattering among all methods for all land cover types and both frequency bands. Moreover, the three general methods yield higher double-bounce scattering than Y4R method, which could be attributed to the building targets inside the park. For park at L-band, double-bounce scattering is stronger and becomes dominating in all three general methods, while a dominating volume scattering is still observed in Y4R. It indicates that radar frequency results in differences on the scattering interpretation when using polarimetric SAR model-based decomposition techniques, and the general method is able to detect this difference. In the largely oriented Building A area, the three general methods present a dominant double-bounce scattering. The volume scattering in Y4R is relatively high in comparison with the other methods at two radar frequency, while L-band with longer wavelength could reduce the volume scattering a little. In Building B area, all methods show a dominant double-bounce scattering at $\mathrm{C}$ - and L-band, and the volume scattering decreases a little at L-band. In the ocean area, all methods consistently show much higher surface scattering, as expected.

Consistent with the simulation results, it can be seen in Table II and III that the proposed GMD-SNVSM overall presents values closer to GMD-GVSM, even though there are relatively large differences in Building A and Building B areas. From theoretical analysis, according to equation (5), the coherency matrix of SNVSM corresponds to $T 22=T 33$ (random volume) for $\tau>1 / 2$ and $T 22 \neq T 33$ (non-random volume) for $\tau \leq 1 / 2$. However, the coherency matrix of GVSM always holds the condition of $T 22=T 33$ (see equation (10)). Therefore, theoretically the GMD-SNVSM method will present a larger difference in decomposition results with the GMD-GVSM method wherever $\tau \leq 1 / 2$. The corresponding values of $\tau$ from GMD-GVSM method at C- and L-band are shown in Fig. 4(c) and (d), respectively. It can be seen that $\tau$ reaches higher values in forest, park and ocean areas with steep incidence angles, whereas lower values appear in built-up and ocean areas with shallow incidence angle. For further understanding, the percentage of $\tau$ values derived from GMD-SNVSM method for different land cover patches are shown in Table IV. It can be seen that there are $99 \%$ pixels on average in Forest, Park and Ocean area with $\tau>1 / 2$. It means that the ocean area with steep incidence angle and two selected vegetation-covered areas in the scene almost fulfill the random volume condition, i.e. T22=T33. Therefore, the proposed SNVSM shows high consistence with GVSM. On the contrary, there are up to $10 \%$ pixels in Building A and Building $\mathrm{B}$ areas with $\tau \leq 1 / 2$, i.e., non-random volume case. Therefore, relatively large decomposition differences appear in these areas. These interpretation results are consistent with the general consideration of vegetation as a random volume and building areas as non-random volumes. In addition, GMD-SNVSM presents a larger volume scattering component than GMD-GVSM since Pv at most reaches a larger value with $4\left\langle\left|S_{h v}\right|^{2}\right\rangle / \tau$ for $\tau \leq 1 / 2$. Although it is still hard to decide which model behaves best from the average scattering power proportion of each component, the results show the potential advantage of SNVSM in distinguishing more volume cases, which needs further investigation. The computation time of GMD-SNVSM is comparable with GMD-GVSM because they entail only one time-consuming nonlinear optimization process, and they are significantly faster than the Modified Chen method which needs to run four times the nonlinear optimization process.

\section{CONCLUSION}

A modified general polarimetric model-based decomposition method using the simplified Neumann generalized volume scattering model (i.e. GMD-SNVSM) is proposed in this letter. In this method, the volume is chosen according to two types of SNVSM for horizontal dipoles or vertical dipoles, respectively, which cover a wide range of volume scattering types. Monte Carlo simulations tests indicate that the proposed GMD-SNVSM method outperforms the original approach and shows consistence with other existing general volume scattering models (i.e. GMD-GVSM). In addition, two real data tests 
using fully polarimetric C-and L-band AIRSAR images over San Francisco region show that the proposed GMD-SNVSM method produces reasonably physical results, as GMD-GVSM method, and outperforms the traditional Y4R method. The different performances of SNVSM and GVSM in two building areas show the potential of SNVSM in identifying more volume cases than GVSM. Directly solving all parameters of generalized volume scattering models, in case of more observations are available, needs further investigation. In addition, testing this method in complex vegetated areas and making further quantitative analysis and comparisons are pending tasks.

TABLE II

AVERAGE SCATtERING POWER PROPORTIONS FOR DIFFERENT LAND COVER PATCHES WITH C-BAND AIRSAR IMAGE

\begin{tabular}{cccccc}
\hline \hline Area & Methods & Ps(\%) & Pd(\%) & Pv(\%) & Pc(\%) \\
\hline \multirow{5}{*}{ Forest } & Y4R & 29.94 & 11.40 & 51.32 & 7.35 \\
& Modified Chen & 30.65 & 21.69 & 40.32 & 7.34 \\
& GMD-GVSM & 28.52 & 21.35 & 42.77 & 7.36 \\
& GMD-SNVSM & 28.33 & 21.37 & 42.94 & 7.36 \\
\hline \multirow{5}{*}{ Park } & Y4R & 25.76 & 25.96 & 42.30 & 5.98 \\
& Modified Chen & 24.59 & 35.22 & 34.22 & 5.97 \\
& GMD-GVSM & 23.31 & 34.77 & 35.94 & 5.98 \\
& GMD-SNVSM & 23.13 & 34.75 & 36.14 & 5.98 \\
\hline \multirow{5}{*}{ Build-up A } & 41.91 & 21.61 & 30.19 & 6.28 \\
& Y4R & 35.25 & 40.98 & 17.61 & 6.16 \\
& GMD-GVSM & 35.38 & 40.35 & 18.12 & 6.16 \\
& GMD-SNVSM & 35.27 & 39.47 & 19.10 & 6.16 \\
\hline \multirow{5}{*}{ Build-up B } & Y4R & 19.10 & 59.86 & 18.28 & 2.76 \\
& Modified Chen & 14.15 & 67.44 & 15.66 & 2.75 \\
& GMD-GVSM & 14.62 & 65.34 & 17.29 & 2.76 \\
& GMD-SNVSM & 14.45 & 64.71 & 18.08 & 2.76 \\
\hline \multirow{5}{*}{ Ocean } & Y4R & 92.36 & 1.93 & 4.64 & 1.07 \\
& Modified Chen & 90.13 & 5.37 & 3.43 & 1.07 \\
& GMD-GVSM & 89.70 & 4.95 & 4.28 & 1.07 \\
& GMD-SNVSM & 89.54 & 4.95 & 4.44 & 1.07 \\
\hline \hline
\end{tabular}

TABLE III

AVERAge SCATtERING POWER PROPORTIONS FOR DiFFERENT LAND COVER PATCHES WITH L-BAND AIRSAR IMAGE

\begin{tabular}{|c|c|c|c|c|c|}
\hline Area & Methods & $\operatorname{Ps}(\%)$ & $\operatorname{Pd}(\%)$ & $\operatorname{Pv}(\%)$ & Pc(\%) \\
\hline \multirow{4}{*}{ Forest } & Y4R & 27.81 & 18.59 & 44.92 & 8.68 \\
\hline & Modified Chen & 27.36 & 29.30 & 34.66 & 8.68 \\
\hline & GMD-GVSM & 26.20 & 28.67 & 36.43 & 8.70 \\
\hline & GMD-SNVSM & 26.02 & 28.64 & 36.64 & 8.70 \\
\hline \multirow{4}{*}{ Park } & $\mathrm{Y} 4 \mathrm{R}$ & 29.43 & 29.20 & 34.71 & 6.66 \\
\hline & Modified Chen & 24.93 & 40.21 & 28.21 & 6.65 \\
\hline & GMD-GVSM & 24.67 & 39.50 & 29.16 & 6.67 \\
\hline & GMD-SNVSM & 24.46 & 39.39 & 29.48 & 6.67 \\
\hline \multirow{4}{*}{ Building A } & Y4R & 30.99 & 37.60 & 24.74 & 6.67 \\
\hline & Modified Chen & 27.22 & 49.62 & 16.59 & 6.57 \\
\hline & GMD-GVSM & 27.34 & 49.11 & 16.96 & 6.59 \\
\hline & GMD-SNVSM & 27.19 & 48.51 & 17.70 & 6.70 \\
\hline \multirow{4}{*}{ Building B } & $\mathrm{Y} 4 \mathrm{R}$ & 21.41 & 59.42 & 16.10 & 3.07 \\
\hline & Modified Chen & 15.53 & 68.22 & 13.19 & 3.06 \\
\hline & GMD-GVSM & 16.25 & 66.32 & 14.37 & 3.06 \\
\hline & GMD-SNVSM & 15.95 & 65.69 & 15.29 & 3.07 \\
\hline \multirow{4}{*}{ Ocean } & $\mathrm{Y} 4 \mathrm{R}$ & 93.85 & 1.86 & 3.52 & 0.77 \\
\hline & Modified Chen & 91.82 & 5.35 & 2.06 & 0.77 \\
\hline & GMD-GVSM & 91.71 & 5.31 & 2.21 & 0.77 \\
\hline & GMD-SNVSM & 91.68 & 5.31 & 2.24 & 0.77 \\
\hline
\end{tabular}

TABLE IV

PERCENTAGE OF PIXELS WITH THE TWO $\tau$ INTERVALS FOR DIFFERENT PATCHES

\begin{tabular}{ccccccc}
\hline \hline \multirow{2}{*}{ Band } & \multirow{2}{*}{$\tau$} & Forest & Park & $\begin{array}{c}\text { Building } \\
\text { A }\end{array}$ & $\begin{array}{c}\text { Building } \\
\text { B }\end{array}$ & Ocean \\
\hline \multirow{2}{*}{$\mathrm{C}$} & $\tau>1 / 2$ & 99.94 & 99.42 & 91.37 & 91.96 & 99.99 \\
\cline { 2 - 7 } & $\tau \leq 1 / 2$ & 0.06 & 0.58 & 8.63 & 8.04 & 0.01 \\
\hline \multirow{2}{*}{$\mathrm{L}$} & $\tau>1 / 2$ & 99.62 & 98.84 & 92.94 & 89.90 & 1 \\
\cline { 2 - 7 } & $\tau \leq 1 / 2$ & 0.38 & 1.16 & 7.06 & 10.10 & 0 \\
\hline \hline
\end{tabular}

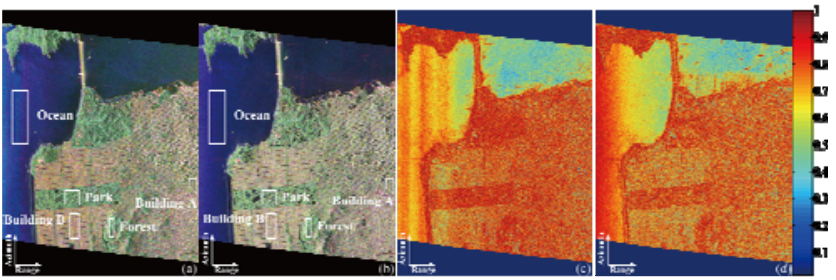

Fig. 4. GMD-SNVSM Decomposition results and corresponding $\tau$ values at C-band (a)\&(c) and L-band (b)\&(d) AIRSAR data. The images are colored by Pd (red), Pv (green), Ps (blue).

\section{REFERENCES}

[1] S. R. Cloude and E. Pottier, "A review of target decomposition theorems in radar polarimetry," IEEE Trans. Geosci. Remote Sens., vol. 34, no. 2, pp. 498-518, Mar. 1996.

[2]J. S. Lee and E. Pottier, Polarimetric Radar Imaging: From Basics to Applications. Boca Raton, FL: CRC Press, 2009.

[3]S. R. Cloude, Polarisation Applications in Remote Sensing. Oxford Univ. Press, 2009

[4] S. W. Chen, Y. Z. Li, X. S. Wang, S. P. Xiao, and M. Sato, "Modeling and Interpretation of Scattering Mechanisms in Polarimetric Synthetic Aperture Radar: Advances and perspectives," IEEE Signal Process. Mag., vol. 31, no. 4, pp. 79-89, Jul. 2014.

[5] A. Freeman and S. L. Durden, "A three-component scattering model for polarimetric SAR data," IEEE Trans. Geosci. Remote Sens., vol. 36, no. 3, pp. 963-973, May 1998.

[6]S. W. Chen, X. S. Wang, S. P. Xiao, and M. Sato, "General Polarimetric Model-Based Decomposition for Coherency Matrix," IEEE Trans. Geosci. Remote Sens., vol. 52, no. 3, pp. 1843-1855, Mar. 2014.

[7] D. Xiang, Y. Ban, and Y. Su, "Model-Based Decomposition With Cross Scattering for Polarimetric SAR Urban Areas," IEEE Geosci. Remote Sens. Lett., vol. 12, no. 12, pp. 2496-2500, Dec. 2015.

[8] D. Xiang, W. Wang, T. Tang, and Y. Su, "Multiple-component polarimetric decomposition with new volume scattering models for PolSAR urban areas," IET Radar, Sonar Navig., vol. 11, no. 3, pp. 410-419, Mar. 2017.

[9]Q. H. Xie, J. D. Ballester-Berman, J. M. Lopez-Sanchez, J. J. Zhu, and C. C. Wang, "Quantitative Analysis of Polarimetric Model-Based Decomposition Methods," Remote Sens., vol. 8, no. 12, pp. 977, Nov. 2016.

[10] M. Arii, J. J. van Zyl, and Y. Kim, "A General Characterization for Polarimetric Scattering From Vegetation Canopies," IEEE Trans. Geosci. Remote Sens., vol. 48, no. 9, pp. 3349-3357, Sep. 2010.

[11] M. Arii, J. J. van Zyl, and Y. Kim, "Adaptive Model-Based Decomposition of Polarimetric SAR Covariance Matrices," IEEE Trans. Geosci. Remote Sens., vol. 49, no. 3, pp. 1104-1113, Mar. 2011.

[12] X. Huang, J. Wang, and J. Shang, "An Integrated Surface Parameter Inversion Scheme Over Agricultural Fields at Early Growing Stages by Means of C-Band Polarimetric RADARSAT-2 Imagery," IEEE Trans. Geosci. Remote Sens., vol. 54, no. 5, pp. 2510-2528, May 2016.

[13] O. Antropov, Y. Rauste, and T. Hame, "Volume Scattering Modeling in PolSAR Decompositions: Study of ALOS PALSAR Data Over Boreal Forest," IEEE Trans. Geosci. Remote Sens., vol. 49, no. 10, pp. 3838-3848, Oct. 2011

[14] M. Neumann, "Remote sensing of vegetation using multi-baseline polarimetric SAR interferometry: Theoretical modeling and physical parameter retrieval," Ph.D. dissertation, Univ. Rennes 1, Rennes, France, Jan. 2009

[15] M. Neumann, L. Ferro-Famil, and A. Reigber, "Estimation of forest structure, ground, and canopy layer characteristics from multibaseline polarimetric interferometric SAR data," IEEE Trans. Geosci. Remote Sens., vol. 48, no. 3, pp. 1086-1104, Mar. 2010.

[16] Q. H. Xie, J. D. Ballester-Berman, J. M. Lopez-Sanchez, J. J. Zhu, and C. C. Wang, "On the Use of Generalized Volume Scattering Models for the Improvement of General Polarimetric Model-Based Decomposition," Remote Sens., vol. 9, no. 2, pp. 117, Jan. 2017.

[17] S. R. Cloude and K. P. Papathanassiou, "Three-stage inversion process for polarimetric SAR interferometry," Proc. Inst. Elect. Eng.-Radar Sonar Navigat., vol. 150, no. 3, pp. 125-134, Jun. 2003. 